\title{
Identidades estudiantiles y retos pedagógicos
}

\section{Students' Identities and Pedagogical Challenges}

\author{
Maureen Camacho Oviedo? \\ División de Educación Básica, Centro de Investigación y Docencia en Educación \\ Universidad Nacional \\ Heredia, Costa Rica \\ mcamac@una.cr \\ Rocío Castillo Cedeño ${ }^{2}$ \\ División de Educación Básica, Centro de Investigación y Docencia en Educación \\ Universidad Nacional \\ Heredia, Costa Rica \\ rociocastillocedeno@gmail.com \\ Heidy León Arce \\ División de Educación Básica, Centro de Investigación y Docencia en Educación \\ Universidad Nacional \\ Heredia, Costa Rica \\ hleon@una.cr
}

1 Máster en Educación con énfasis en Docencia Universitaria, otorgado por la Universidad Nacional, Costa Rica. Licenciada en Ciencias de la Educación con concentración en Educación Básica I y II Ciclos. Actualmente, se desempeña como subdirectora de la División de Educación Básica (DEB) del Centro de Investigación y Docencia en Educación (CIDE) de la Universidad Nacional (UNA) y como responsable del Programa de Desarrollo Académico y Gestión Organizacional de la División de Educación Básica. Como académica de la DEB, en la Carrera de Pedagogía con énfasis en I y II Ciclos de la Educación General Básica, se ha desempeñado en el área de docencia, en los procesos de Intervención Pedagógica a nivel de diplomado y bachillerato, y como responsable de la Carrera de Pedagogía con énfasis en I y II Ciclos. Ha laborado como docente de primaria en centros educativos públicos y privados.

2 Doctora en Educación con mención en Mediación Pedagógica. Magíster en Administración Educativa y en Psicopedagogía. Profesora y bachiller en ciencias de la Educación con énfasis en educación preescolar, Universidad Nacional. Veintiún años de experiencia en el ámbito de la educación preescolar y la universitaria. Ha participado activamente en procesos de formación de formadores, principalmente, en el diseño e implementación de cursos de pedagogía a distancia, en la Universidad Estatal a Distancia. Durante cinco años se desempeño como decana de la Facultad de Educación de la Universidad De La Salle Costa Rica y trabajo durante un año como Vicerrectora Académica en esta misma universidad. Actualmente, se desempeña como docente e investigadora en el Centro de Investigación y Docencia en Educación de la Universidad Nacional de Costa Rica. Autora del libro: La conciencia cósmica en la primera infancia. Reflexiones paradigmáticas, propuestas biopedagógicas y un acercamiento a la espiritualidad de las niñas y los niños.

3 Máster en Pedagogía con énfasis en Diversidad de los Procesos Educativos, Licenciada en Educación Especial con énfasis en Integración, Universidad Nacional de Costa Rica. Educadora, académica e investigadora de la División de Educación Básica del Centro de Investigación y Docencia en Educación (CIDE) de la Universidad Nacional. Actualmente gestora de la Carrera de Educación Especial y participante del Programa Desarrollo Académico. 
URL: http://www.una.ac.cr/educare

CORREO: educare@una.cr

\author{
Alfredo Miranda Calderón ${ }^{4}$ \\ División de Educación Básica, Centro de Investigación y Docencia en Educación \\ Universidad Nacional \\ Heredia, Costa Rica \\ alfremira@gmail.com \\ Zulay Pereira Pérez ${ }^{5}$ \\ División de Educación Básica, Centro de Investigación y Docencia en Educación \\ Universidad Nacional \\ Heredia, Costa Rica \\ zulay.pereira.pérez@una.cr \\ Erika Vásquez Salazar ${ }^{6}$ \\ División de Educación Básica, Centro de Investigación y Docencia en Educación \\ Universidad Nacional \\ Heredia, Costa Rica \\ erika.vasquez.salazar@una.cr
}

Recibido 26 de setiembre de 2012 - Corregido 04 de febrero de 2013 • Aceptado 13 de marzo de 2013

4 Académico en la División de Educación Básica del Centro de Investigación y Docencia en Educación de la Universidad Nacional, Costa Rica. Magíster con especialidades en Administración Educativa, Universidad de Costa Rica y Formación de Formadores de Docentes de Educación Primaria, Universidad Nacional. Bachiller en Actuación y Dirección de las Artes Dramáticas y en Ciencias de la Educación en I y II Ciclos-UCR. Licenciado en Ciencias de la Educación en I y II ciclos con énfasis en Educación Rural. Como maestro rural unidocente ha implementado modelos de gestión participativa, y diversas investigaciones y exploraciones relacionadas con el aprendizaje mediado por el uso y aprovechamiento del entorno natural. Maestro en la Escuela Nueva Laboratorio Emma Gamboa UCR. Actualmente es gestor académico de la carrera de Pedagogía con énfasis en I y II Ciclos. DEB- Autor de diversos artículos relacionados con la educación en contextos rurales, arte en la mediación pedagógica e investigación. Ha liderado procesos de capacitación docentes de educación primaria. Ponente en congresos nacionales e internacionales.

5 Doctora en Educación, de la Universidad Estatal a Distancia. Catedrática de la Universidad Nacional. Máster y Licenciada en Psicología, de la Universidad de Costa Rica. Adscrita al Colegio Profesional de Psicólogos de Costa Rica. Ex coordinadora del Programa de Investigación en Epistemología Genética y Educación del IIMEC (INIE)UCR. Ex Directora de la División de Educación Básica de la Universidad Nacional. Investigadora y docente del Centro de Investigación y Docencia en Educación (CIDE-UNA). Tiene publicaciones en el campo de la psicología y de la pedagogía. Autora del libro: La mirada estudiantil hacia las características docentes: las dinámicas interactivas, los rasgos docentes y el clima de aula universitario analizados con un diseño de método mixto.

6 Académica en la División de Educación Básica del Centro de Investigación y Docencia en Educación de la Universidad Nacional, Costa Rica. Máster con especialidad en Pedagogía de la Universidad Nacional. Bachiller en Ciencias de la Educación I y II ciclo concentración Inglés de la Universidad Estatal a Distancia. Licenciada en Docencia de la Universidad Estatal a Distancia. Actualmente es gestora de la carrera de Enseñanza del Inglés para I y II Ciclos. 
Resumen. El presente artículo, surge del proceso de inducción de estudiantes de primer ingreso, en el 2012, a cuatro carreras de Pedagogía en la División de Educación Básica del Centro de Investigación y Docencia en Educación (CIDE) en la Universidad Nacional. Recoge la información de 110 estudiantes. Busca la identificación de rasgos que permitan la conformación de identidades estudiantiles y los posibles retos pedagógicos que de ello se deriven. Retoma las acciones llevadas a cabo para el diseño y ejecución del proceso de inducción y los datos que aportan los instrumentos utilizados. Los hallazgos acerca de las identidades estudiantiles plantean áreas fuertes y áreas por desarrollar, que, en este caso particular, se convierten en los retos pedagógicos que han de ser atendidos desde la unidad académica. La División de Educación Básica, formadora de profesionales en diferentes disciplinas, tiene una gran responsabilidad en la transformación social y cultural del país, preparando profesionales en educación y brindando las herramientas necesarias para que los estudiantes y las estudiantes puedan lograr mayores habilidades artísticas, habilidades en redacción y ortografía, habilidades tecnológicas, habilidades deportivas, habilidades para hablar en público: estos son algunos de los retos que emergen del análisis del proceso de inducción realizado con estudiantes de nuevo ingreso.

Palabras claves. Pedagogía, identidades estudiantiles, retos pedagógicos, universidad, estudiantes, primer ingreso, inducción, Revista Electrónica Educare, Costa Rica.

Abstract. This article is a result from the orientation process carried out for the first-year students of four study programs of the Basic Education Division at the Center for Research and Teaching in Education (CIDE), Universidad Nacional, Costa Rica, in 2012. Information from 110 students was collected and analyzed with the purpose to identify the students' identity traits and possible pedagogical challenges associated. The actions taken during the design and execution of the orientation process and the data collected through different instruments were taken into account for the analysis. The findings include strong areas, as well as areas "to be developed" which represent the pedagogical challenges of the academic unit. The Basic Education Division (or School of Basic Education) plays a fundamental role in the country's social and cultural transformation preparing professionals in Education and providing students with the necessary tools for developing better skills in areas such as art, writing, technology, sports, and public speaking. These are some of the challenges emerging from the analysis of the orientation process for first-year students.

Keywords. Pedagogy, students' identities, pedagogical challenges, university, students, first-year, orientation, Educare Electronic Journal, Costa Rica.

\section{Introducción}

La División de Educación Básica (DEB), a partir de una iniciativa denominada Formación de Formadores, dio origen a un programa permanente a partir del 2002 con el propósito de fortalecer a los académicos, académicas, y al estudiantado. Dicho programa se reformula en el 2011 con el nombre de Programa Desarrollo Académico y Gestión Organizacional (Camacho et al., 2011a) considerando además los resultados aportados por el Informe Final de Desarrollo Académico 2006-2010 (Camacho, Espinoza et al., 2012). Este se constituye como una estructura dinámica y compleja que pretende la promoción del pensamiento y el establecimiento de líneas de acción, en busca de la planificación de la gestión académica-administrativa de la unidad académica. Existe una permanente preocupación por la promoción y actualización de las carreras y, para ello, se ha recurrido a la figura del gestor y gestoras como personas que median los procesos de desarrollo en los que participan los académicos y académicas en general. 
URL: http://www.una.ac.cr/educare

CORREO: educare@una.cr

Dentro de las distintas acciones e indicadores que se han propuesto para el adecuado desarrollo de la unidad académica, desde hace varios años, se identifica el proceso de inducción de estudiantes, que se constituye en un indicador de logro congruente con lo planteado en el objetivo general del programa, que a la letra señala:

Fortalecer el desarrollo académico y la gestión organizacional de la Unidad, para la promoción y actualización de las carreras, proyectos de docencia, de investigación, de extensión e integrados, así como la optimización profesional de académicos, administrativos y estudiantes de la División de Educación Básica. (Camacho et al., 2011a, p. 6)

Por ello, la DEB tiene como actividad permanente, al inicio de cada curso lectivo, realizar el "Proceso de Inducción a Estudiantes de Primer Ingreso", en sus cuatro carreras: Pedagogía con énfasis en I y II Ciclos de la Educación General Básica, Pedagogía con énfasis en Educación Preescolar, Educación Especial con énfasis en Integración y la Enseñanza del Inglés en I y ll Ciclos. Dicho proceso es coordinado por la subdirección de la DEB, en colaboración con sus gestores y gestoras de carreras, quienes forman parte del programa mencionado. Con este actividad, primordialmente, se busca acercar al estudiantado de nuevo ingreso, a las autoridades, profesores y profesoras, así como a los distintos servicios que les ofrece la Universidad en general y la Unidad Académica en particular.

Desde el enfoque humanista que caracteriza a la Universidad Nacional (UNA), el proceso de inducción se visualiza no solamente, como un momento inicial aislado del proceso, sino como una experiencia pedagógica para acercarse al estudiantado con el propósito de hacerlo sentir parte de la universidad, comunicándoles que la razón de ser de esta son ellos y ellas y que sus necesidades e intereses son importantes.

También, brinda la posibilidad de motivar hacia el reconocimiento de valores y principios que caracterizan a la institución universitaria:

La Universidad Nacional está comprometida con un ideal de sociedad donde prevalezcan los principios de justicia, equidad y libertad; una sociedad donde exista igualdad de oportunidades, donde se promueva el desarrollo de todos los sectores del país en un sentido integral. (Universidad Nacional Costa Rica, 2005, p. 6)

Considerando los principios resaltados en el Modelo Pedagógico de la Universidad Nacional, el proceso de inducción también responde a lo siguiente:

- Mejoramiento continuo en la formación integral de los estudiantes y los procesos de gestión académica-administrativa y paraacadémica.

- Visión prospectiva que permite la planificación estratégica para el logro de objetivos a mediano y largo plazo. (Universidad Nacional Costa Rica, 2005, p. 5) 
Para el curso lectivo 2012, las personas integrantes del Programa de Desarrollo Académico y Gestión Organizacional, con apoyo de colaboradoras, establecieron mecanismos con el fin de organizar y hacer del proceso una experiencia positiva para los cuatro grupos de estudiantes de nuevo ingreso. Con el acercamiento al nuevo grupo de estudiantes (110 alumnos y alumnas) y mediante la recopilación de información a partir de un cuestionario aplicado a todos, surge este documento, con el propósito de reflejar las identidades estudiantiles y los retos pedagógicos que plantea dicho conocimiento. Una vez determinados los principales rasgos y aspectos que caracterizan las particularidades de cada estudiante de los respectivos grupos por carrera, se procedió a diseñar una serie de acciones y actividades conducentes al logro de dos grandes propósitos: desarrollar el sentido de pertenencia e identidad con su instancia formadora, así como apoyar y fortalecer su desempeño académico mediante un adecuado acompañamiento en esta etapa de su formación. En estas páginas, se expondrán los principales logros y hallazgos generados de este proceso, así como las implicaciones y retos que la actual coyuntura educativa plantea a la formación docente y a las diversas instancias de formación universitaria. Una profunda reflexión en torno a esta experiencia académica se hace necesaria como parte del quehacer y misión de la División de Educación Básica.

\section{Contextualización y metodología}

El proceso de inducción desarrollado en el 2012 en la División de Educación Básica (Camacho et al., 2012), en el marco del Programa DAGO, requirió del diseño e implementación de una serie de instrumentos y coordinaciones logísticas para su respectiva aplicación al estudiantado. Estas se describen a continuación:

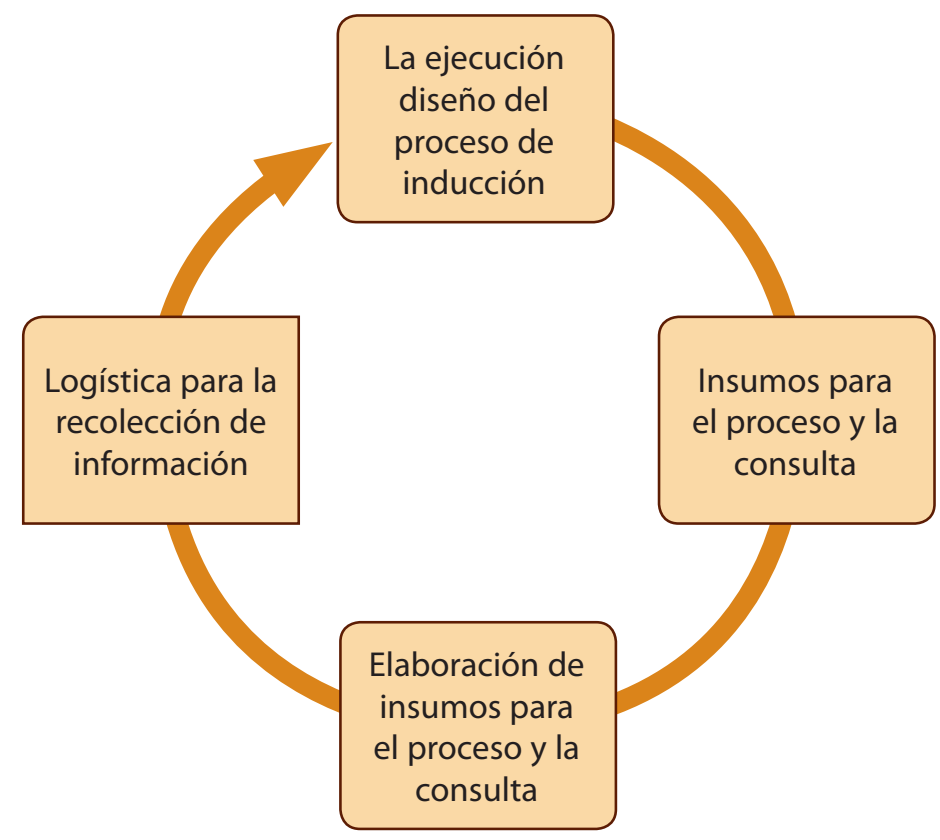

Figura 1: Fases del proceso de inducción a estudiantes de primer ingreso 
URL: http://www.una.ac.cr/educare

CORREO: educare@una.cr

A. Diseño del proceso de inducción: se elaboraron algunos documentos para guiar el proceso. En primer lugar, se diseñó una agenda general para el proceso de inducción que contemplaba las distintas acciones a realizar: Presentación de las autoridades de la DEB y palabras de bienvenida; proyección de fondo de video sobre el quehacer de la DEB; motivación por parte de una estudiante del nivel de licenciatura; presentación de la página web de la DEB; participación de los funcionarios del Centro de Información y Documentación de Niñez, Adolescencia y Familia (CIDENAF), en cuanto a servicios y uso de catálogo; presentación y promoción del Programa Éxito Académico de la Universidad Nacional; promoción de la Oficina de Atención Estudiantil; dinámica de integración; refrigerio y conversatorio con gestores de carrera.

B. Insumos para el proceso y la consulta: de igual manera, se diseñó otra agenda, para guiar la realización de un conversatorio con el gestor y gestoras de carrera, quienes son los encargados de la conducción, desarrollo y fortalecimiento de las distintas carreras que se imparten en la DEB y quienes más directamente tienen conexión con el estudiantado. Dicho conversatorio, contempló diversas acciones: Saludo y motivación, entrega y revisión de material; control de asistencia; llenado de la ficha del estudiante; llenado de la malla curricular; nombramiento de un representante o una representante de grupo; y distribución para la realización del conversatorio.

Paralelo a lo anterior, el equipo del PDAGO, diseñó una ficha que recopiló los principales rasgos estudiantiles y que permitió lograr definir su identidad como grupo de primer ingreso a las carreras de la DEB para el 2012.

C. Logística para la recolección de información: por otra parte, se preparó una carpeta para el estudiantado con una serie de materiales para utilizar con ellos y ellas, y visualizar su organización académica. Se les ofreció, de igual manera, información relevante para su apropiado desempeño en la Unidad Académica y organización con referencia al plan de estudios de la carrera que cursan. Dentro de los materiales, estuvieron un desplegable de la DEB con una reseña histórica de la DEB, su visión; lo que ofrece como Unidad Académica; horarios de atención, teléfonos para acceder; así como el listado con el nombre y correos electrónicos del gestor y gestoras de las carreras. Además, la malla curricular del plan de estudios de cada una de las respectivas carreras, información de la Oficina de Atención Estudiantil; también, a manera de estímulo, se les entregó un ejemplar de la revista EDUCARE, un lápiz y lapicero.

El equipo organizador coordinó, además, un espacio para la participación de otros programas y servicios que ofrece la Universidad y la Unidad Académica (Página web de la DEB, CIDENAF, Éxito Académico, Oficina de Atención Estudiantil).

D. La ejecución: la culminación del proceso de inducción se llevó a cabo el día jueves $1^{\circ}$ de marzo de 2012, a la 1:00 p.m., en el auditorio "Marco Tulio Salazar" y en aulas del Centro de Investigación y Docencia en Educación (CIDE), para los estudiantes y las estudiantes de primer ingreso de las cuatro carreras de la Unidad Académica.

Se siguió la agenda propuesta en el espacio de tiempo que se había estimado, con el desarrollo apropiado de todas las actividades, en cuanto a la actividad recreativa, la cual se desarrolló 
con la totalidad de participantes en el mismo auditorio. Posterior a ello, se ofreció el refrigerio y luego el estudiantado pasó con el gestor y gestoras de la carrera correspondiente para el desarrollo del conversatorio y la recopilación de la información requerida para su conocimiento y posterior apoyo.

Se partió de la idea de que la actividad no solo habría de constituirse en una instancia para que los estudiantes y las estudiantes de primer ingreso conocieran la dinámica universitaria, sino, prioritariamente, para que la unidad académica fuera consciente de las identidades estudiantiles, sus rasgos más significativos y, a partir de ello, pudiera organizarse en distintos ámbitos para responder a los retos pedagógicos que dichas identidades le demandaran.

\section{Identidades estudiantiles}

La identidad de los estudiantes y las estudiantes se construye de una forma cuidadosa y compleja. Esta se cimienta, de acuerdo con Venegas (2009), de las imágenes del pasado, de recuerdos de la escuela, de la formación, estereotipos, demandas, medios de comunicación, de los hogares y de los centros educativos. Todo esto lleva al estudiantado a cumplir con ciertas funciones sociales dentro de los procesos de enseñanza y aprendizaje.

Los procesos de enseñanza-aprendizaje se ven influidos por múltiples factores, algunos de ellos circunscritos al ámbito personal, socioemocional y educativo. De modo que si se desea fortalecer el desarrollo del estudiante de manera integral, se han de retomar los planteamientos de Ocampo (2007), quien concibe al ser humano desde las dimensiones mente, corazón y voluntad. De ahí que pueda afirmarse que un reto pedagógico es la búsqueda de alternativas que favorezcan el desarrollo de los estudiantes y las estudiantes en sus distintas áreas. En ese sentido, es valioso, según nuestro criterio, conocer las identidades estudiantiles que sirvan de guía para los planteamientos pedagógicos en busca de opciones para el desarrollo del estudiantado como persona individual y social.

Guevara (2009) concibe la universidad como:

La universidad es un espacio educativo en el que se gestan vínculos de diversa índole que configuran una trama de elementos necesarios de ser internalizados por los estudiantes y las estudiantes. Allí comienza un itinerario educativo en el que se entrecruzan múltiples elementos: la trama de creencias, los valores, los principios, ritmos de aprendizaje, condiciones sociales específicas, géneros y procedencia diversa, variables que dotan de elevada complejidad a la práctica estudiantil (...). (p. 219)

Agrega la autora que, en ese proceso de hacerse estudiante, se van estableciendo variadas relaciones con el conocimiento y entretejidos sociales a partir de la interacción con los pares. En esa dinámica estudiantil, se van generando vínculos de afecto, dificultades y en el diario vivir, es cuando realmente se va construyendo la cultura estudiantil. 
URL: http://www.una.ac.cr/educare

CORREO: educare@una.cr

Por otra parte, Pereira (2010) señala que la educación como valor social debe ser un medio mediante el cual se promueva la formación del estudiantado no solo desde la perspectiva cognoscitiva, sino también desde la humanización del ser, siempre con la mirada en el objetivo de que su inserción como futuros profesionales esté acorde con los cambios vertiginosos de la sociedad, y donde se considere, también, la emoción y el sentimiento como factores constitutivos de la formación. De ahí que el reconocer las identidades estudiantiles se constituya en un aspecto indispensable para atender las necesidades de formación del estudiantado con miras a un futuro más sano y eficaz.

En el sentido señalado, parece pertinente asumir las identidades estudiantiles como un reto pedagógico, donde cobre realmente sentido la realización de un proceso de inducción que tenga pertinencia pedagógica y que enrumbe las acciones por desarrollar con los distintos grupos estudiantiles. Si se parte del hecho de que cada cohorte tiene identidades particulares, una historia y una vivencia que es muy propia y que condicionará de alguna manera su relación con la apropiación del conocimiento y con las dinámicas interactivas que se generen en el salón del clase -y de las cuales no están ausentes los docentes y las docentes que les tienen como alumnos y alumnas-, es entonces pertinente hacer un esfuerzo por conocer esos rasgos estudiantiles. A nuestro criterio, también resulta oportuno el establecimiento de acciones tendientes al favorecimiento del aprendizaje y desarrollo integral del estudiantado.

Al respecto, Guevara (2009) argumenta que:

Las características de ese contexto sociocultural novedoso, en interjuego con sus componentes subjetivos, personales, provenientes de la esfera motivacional, delinearán las formas particulares y colectivas del proceso de construcción de identidades estudiantiles, esto es, necesariamente, en articulación con los nuevos elementos que el escenario universitario ofrece. (p. 220)

En esa misma línea de pensamiento, Castells (1997), citado por Guevara (2009), señala que las identidades son significativas para los autores sociales cuando las mismas se internalizan y construyen su significado alrededor de esa internalización.

Es importante notar que en el contexto universitario coexisten tanto identidades particulares, como las identidades estudiantiles que se conforman en el grupo y las cuales estarán marcadas no solo por los rasgos de cada uno de los estudiantes y las estudiantes, sino también por las interacciones que, en el diario convivir, va estableciendo el estudiantado con sus iguales y con el profesorado a cargo. Conocer esas dinámicas, tratar de identificar rasgos básicos, aunque no sea una tarea fácil, ha de constituirse en un elemento importante para la práctica en las aulas. En este caso, aunque se reconoce que identificar algunos de los rasgos estudiantiles representa solo un esbozo de lo que realmente se requiere conocer, es un paso que constituye un primer acercamiento a las identidades estudiantiles, con miras pedagógicas. 
Para la definición de las identidades estudiantiles de primer ingreso en el año 2012 a las carreras de Pedagogía, se utilizó como instrumento para la recolección de datos una ficha estudiantil que recopilaba información acerca de características personales, opciones de carrera, habilidades, principales debilidades, problemáticas diversas que podrían afectarle en sus estudios, entre otros aspectos. Dicho instrumento se aplicó a la totalidad de estudiantes (110 estudiantes) de primer ingreso de las cuatro carreras de pedagogía de la DEB.

En el apartado siguiente se destacan los datos principales que permiten una idea de las identidades estudiantiles de la promoción 2012 a las carreras de la División de Educación Básica.

\section{Rasgos que identifican las identidades estudiantiles}

A continuación se exponen las principales características que definen a los estudiantes y a las estudiantes de primer ingreso de las carreras de la DEB. Se inicia con una caracterización del grupo en función de los intervalos de edad, sexo y estado civil.

Seguidamente, se retoman los datos referentes al disfrute o no de beca universitaria, a las visiones estudiantiles acerca de las áreas fuertes y débiles, así que como a los principales factores que, desde su perspectiva, podrían incidir en sus estudios universitarios.

Tabla 1

Caracterización estudiantil por intervalo de edad, sexo, y estado civil, según carrera en la que se encuentran matriculados

\begin{tabular}{|c|c|c|c|c|}
\hline Criterios & $\begin{array}{l}\text { Pedagogía } \\
\text { I y || Ciclos }\end{array}$ & $\begin{array}{l}\text { Pedagogía con énfasis } \\
\text { en Educación Preescolar }\end{array}$ & Enseñanza del Inglés & $\begin{array}{l}\text { Educación Especial con } \\
\text { énfasis en Integración }\end{array}$ \\
\hline \multicolumn{5}{|c|}{ Intervalos de edad } \\
\hline 17-19 años & 19 & 25 & 19 & 26 \\
\hline 20-22 años & 05 & 03 & 06 & 02 \\
\hline 23-25 años & 01 & - & 01 & - \\
\hline Más de 25 años & 01 & - & - & - \\
\hline \multicolumn{5}{|l|}{ Sexo } \\
\hline Mujeres & 20 & 28 & 19 & 28 \\
\hline Varones & 06 & - & 07 & - \\
\hline \multicolumn{5}{|l|}{ Estado civil } \\
\hline Casado(a) & 01 & - & 01 & - \\
\hline Soltero (a) & 24 & 28 & 25 & 28 \\
\hline Divorciado (a) & - & - & - & - \\
\hline Viudo (a) & - & - & - & - \\
\hline
\end{tabular}


URL: http://www.una.ac.cr/educare

CORREO: educare@una.cr

La tabla 1 permite observar que la mayoría de los estudiantes y las estudiantes de primer ingreso en las cuatro carreras de la DEB, prioritariamente se ubican en orden descendente en los intervalos de edad de 17 a 19 años y de 20 a 22 años, las frecuencias disminuyen en los intervalos de 23 a 25, donde solo se ubica un estudiante de I y II Ciclos y otro de Inglés.

Por otra parte, mayoritariamente, en las cuatro carreras se tiene una población femenina, no obstante cabe destacar que en Pedagogía en I y II ciclos hay matriculados 06 varones y en Enseñanza del Inglés en Pedagogía en I y II Ciclos, 07 varones.

Es importante señalar que, en las cuatro carreras, la condición civil que prevalece en el estudiantado es la soltería y solo un estudiante se reporta como casado en Pedagogía en I y ll Ciclos y otro en Enseñanza del Inglés en Pedagogía en I y II Ciclos, así como uno en unión libre, también, en esta última carrera.

Como puede verse, en las identidades estudiantiles destacan la juventud, el ser mayoritariamente del sexo femenino y el ser solteros y solteras. Razón de más para poder incrementar la atención del estudiantado que, prioritariamente, muestra condiciones que les podrían permitir mayor dedicación al estudio.

De igual manera, se destacan a continuación los datos referidos al disfrute o no de beca otorgada por la Universidad Nacional para apoyar los estudios universitarios de los estudiantes y las estudiantes de primer ingreso en el 2012 en las carreras de la DEB, así como los datos referentes al desempeño laboral del estudiantado.

Tabla 2

Estudiantes que disfrutan de beca y que trabajan, según carrera

\begin{tabular}{llccc}
\hline Carrera & \multicolumn{2}{c}{ Tienen beca } & \multicolumn{2}{c}{ Trabajan } \\
\hline & Fr. & $\%$ & Fr. & $\%$ \\
Pedagogía con énfasis en I y II Ciclos de & 16 & $15 \%$ & 01 & $1 \%$ \\
la Educación General Básica. & & & & \\
Pedagogía con énfasis en Educación Preescolar & 11 & $10 \%$ & 02 & $3 \%$ \\
Educación Especial con énfasis en Integración. & 10 & $9 \%$ & 02 & $3 \%$ \\
Enseñanza del Inglés en I y II Ciclos. & 10 & $9 \%$ & 03 & $8 \%$ \\
\hline Total & 47 & $43 \%$ & 09 & \\
\hline
\end{tabular}

$\mathrm{n}=110$ estudiantes 
La tabla 2 permite observar el disfrute de beca por parte del estudiantado de primer ingreso para las cuatro carreras de la División de Educación Básica. En ese sentido, es importante señalar que solamente un $43 \%$ disfruta de beca y únicamente el $8 \%$ se desempeña en algún tipo de trabajo. Es importante considerar que para muchos y muchas estudiantes, contar con un apoyo económico, puede constituirse en el factor decisivo para mantenerse en la formación universitaria, razón por la cual ese aspecto es fundamental como factor en sus identidades.

De las distintas carreras, resulta pertinente señalar que en Pedagogía con énfasis en I y II Ciclos de la Enseñanza General Básica, el 15\% del estudiantado tiene beca y un 1\% trabaja. En orden descendiente, en Pedagogía con énfasis en Educación Preescolar el 10\% del estudiantado tiene beca y un $2 \%$ trabaja. Por otra parte, en igualdad de condiciones se encuentran las carreras de Educación Especial con énfasis en Integración y Enseñanza del Inglés en I y ll Ciclos, donde el $9 \%$ del estudiantado tiene beca y el 3\% trabaja.

Como puede notarse, la mayoría de los estudiantes y las estudiantes de primer ingreso en las carreras de la división se dedica al estudio y únicamente el $8 \%$ de ellos y ellas combina el estudio con el trabajo. Mientras que menos de la mitad del estudiantado (43\%) disfruta de apoyo mediante una beca universitaria. Esta condición puede ser un elemento de apoyo positivo para promover un sentido de pertinencia, responsabilidad y compromiso de las estudiantes y los estudiantes, hacia las distintas carreras que imparte la unidad académica, así como para desarrollar acciones de mejoramiento dirigidas al estudiantado. Por lo que su atención se constituye en un reto pedagógico impostergable, el hecho de que existe disponibilidad de tiempo hacia el estudio, da margen para que la unidad académica aproveche estas opciones para el desarrollo de alternativas de fortalecimiento para el estudiantado.

Se presentan a continuación, los datos acerca de la opción de carrera con la que ingresó el estudiantado de la promoción 2012 a las carreras de la DEB.

Tabla 3

Opción de carrera con la que ingresaron los estudiantes y las estudiantes a la DEB

\begin{tabular}{lllll}
\hline Carrera & Opción 1 & Opción 2 & Opción 3 & NR \\
\hline $\begin{array}{l}\text { Pedagogía con énfasis en I y II Ciclos de la Educación } \\
\text { General Básica*. }\end{array}$ & 22 & 01 & 01 & - \\
Pedagogía con énfasis en Educación Preescolar & 25 & 02 & 01 & - \\
Educación Especial con énfasis en Integración. & 27 & 01 & - & - \\
Enseñanza del Inglés en I y II Ciclos. & 17 & 08 & 02 & 01 \\
\hline Total & $91(83 \%)$ & $12(11 \%)$ & $04(4 \%)$ & $01(1 \%)$ \\
\hline
\end{tabular}

$\mathrm{n}=110$ estudiantes

* Dos estudiantes (02) cursan Diplomado como segunda opción de carrera. 
URL: http://www.una.ac.cr/educare

CORREO: educare@una.cr

A partir de la tabla 2, se señala que de la totalidad de estudiantes participantes en el proceso de inducción 2012, un 95\% [104 estudiantes], manifiestan encontrarse en la carrera de su preferencia, únicamente un 5\% [05 estudiantes] indican no estar en la carrera de su preferencia y 01 estudiante no dio respuesta.

Contar con estudiantes que, en su mayoría (91\%), se encuentran cursando la carrera de su preferencia como primera opción constituye un factor positivo para las carreras de la División de Educación Básica; no obstante, es importante considerar la situación de estudiantes que tienen la carrera como segunda opción (12 estudiantes, $11 \%$ ), y aunque en menor cantidad en tercera (04 estudiantes, $4 \%$ ), pues en la medida en que el estudiantado se encuentre poco satisfecho o comprometido con una opción de carrera, su nivel de compromiso y dedicación no suele ser muy alto. De ahí que sería importante considerar acciones tendientes a la búsqueda de un nivel de identidad y de ilusión hacia la carrera que están cursando.

\section{Identificación de habilidades y debilidades desde la perspectiva estudiantil}

De igual manera, se hizo una valoración de habilidades y debilidades desde la perspectiva estudiantil y se exponen a continuación los principales resultados obtenidos.

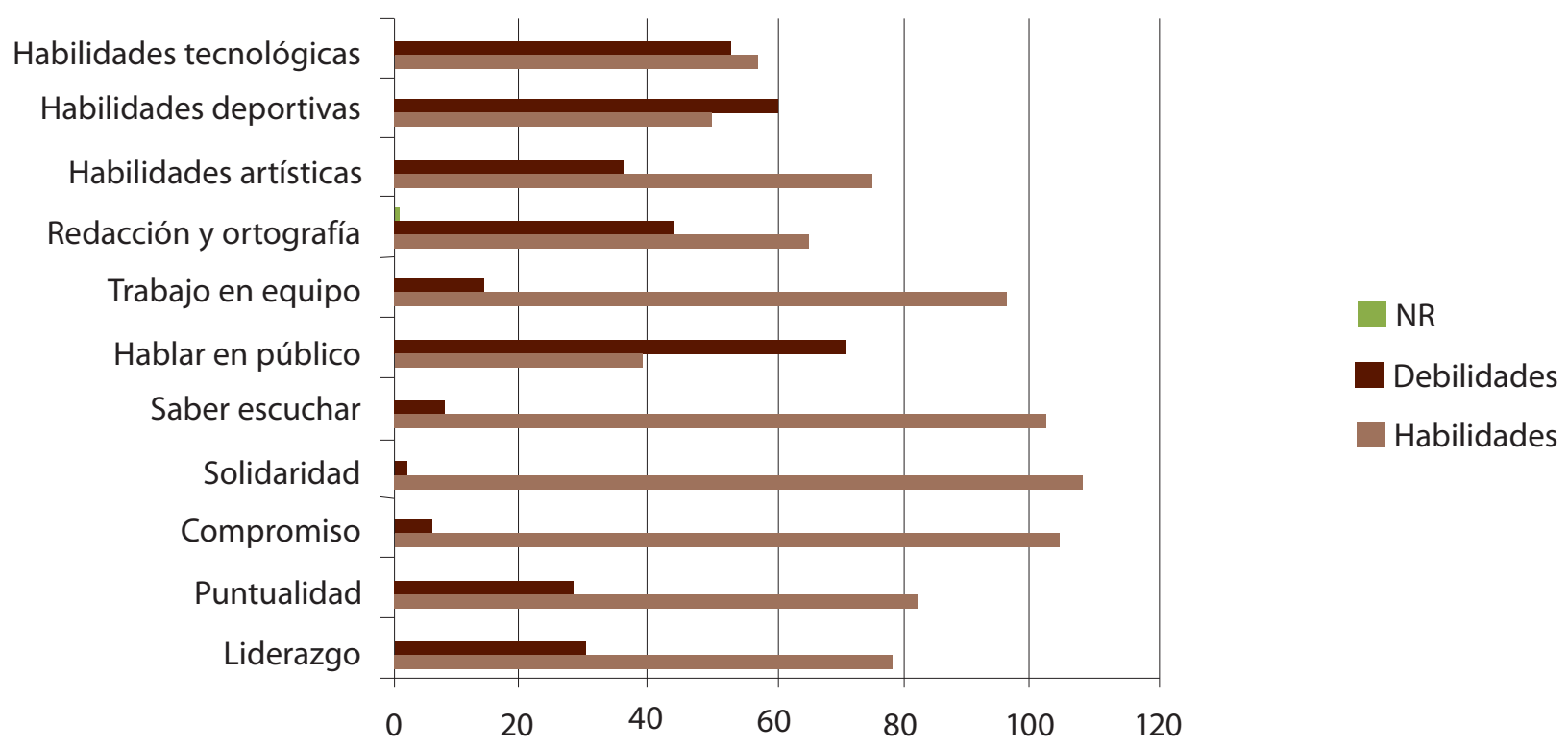

Figura 2. Habilidades y debilidades que poseen los estudiantes y las estudiantes de primer ingreso, según sus propias valoraciones 
La figura 2 muestra la percepción estudiantil de las habilidades y debilidades que poseen. En ese sentido, es importante notar que en orden descendente, la principal habilidad que manifiestan tener es la solidaridad (98\%), seguida por el compromiso (95\%) y el saber escuchar (93\%), trabajo en equipo (87\%), la puntualidad (75\%) y el liderazgo (71\%).

Es importante destacar que las habilidades mencionadas por los estudiantes y las estudiantes de las carreras de la DEB, las siguientes muestran los menores porcentajes: habilidades artísticas (68\%), redacción y ortografía (59\%), habilidades tecnológicas (52\%). habilidades deportivas (45\%), hablar en público (35\%).

Es importante que estas habilidades que obtuvieron bajos porcentajes sean desarrolladas por el estudiantado, pues todas tienen incidencia en su práctica profesional docente. Como se verá a continuación, algunas de las habilidades que tienen menor porcentaje coinciden con las que los estudiantes y las estudiantes argumentan como sus principales debilidades.

Al respecto, al buscar la opinión estudiantil acerca de las que consideran como sus principales debilidades, los resultados indican en orden prioritario las siguientes: hablar en público (65\%), habilidades deportivas (55\%), habilidades tecnológicas (48\%), redacción y ortografía (40\%, no responde 1\%), habilidades artísticas (32\%), liderazgo (27\%, no responde $2 \%)$, puntualidad (25\%), trabajo en equipo (13\%), saber escuchar (8\%), compromiso (6\%), solidaridad (2\%), no responden (2\%).

Es importante destacar que como principales debilidades el estudiantado mencionó el hablar en público, las habilidades deportivas y tecnológicas, así como la redacción y ortografía, todos con porcentajes superiores al 40\%. Estas debilidades están altamente relacionadas con su futura labor como profesionales en el campo educativo, razón por la cual es evidente la necesidad de que se les brinde atención.

\section{Retos pedagógicos}

De manera general puede decirse, que valorando el proceso de inducción realizado en el primer ciclo lectivo, ese fue pertinente y cumplió con las expectativas del equipo del Programa de Desarrollo Académico y Gestión Organizacional de la División de Educación Básica. De igual manera, la participación de las distintas instancias de la Universidad Nacional fue la que se esperaba y brindó, a los participantes y las participantes, la información requerida para comprender los distintos servicios y programas que les ofrece la universidad y la unidad académica.

La determinación de las identidades estudiantiles permite identificar algunos aspectos que se constituyen en retos pedagógicos para el ofrecimiento de condiciones de enseñanzaaprendizaje favorables para el desarrollo integral de los estudiantes y las estudiantes que han ingresado en el presente año a las carreras en pedagogía que ofrece la División de Educación Básica. En ese sentido, cabe señalar como principales retos, los que a continuación se destacan: 
URL: http://www.una.ac.cr/educare

CORREO: educare@una.cr

- Los estudiantes y las estudiantes de la cohorte del 2012, son jóvenes. La mayoría son mujeres, son solteros y solteras y prioritariamente se dedican al estudio, condición que ha de ser aprovechada para fortalecer su desarrollo.

- Optaron en primer lugar por carreras de Pedagogía, lo cual constituye una fortaleza para el futuro trabajo a realizar con ellos y ellas.

- Es bajo el porcentaje de estudiantes de carreras de Pedagogía que disfrutan de beca universitaria.

Los aspectos destacados anteriormente permiten reflexionar acerca de los retos inmediatos que el programa visualiza para dar respuesta a las identidades estudiantiles del grupo de primer ingreso en el 2012. Es innegable que la DEB debe asumir un rol activo en el seguimiento de los estudiantes y las estudiantes, de manera que los procesos de inducción realizados cobren sentido para dichos estudiantes.

Así, por ejemplo, se determinó que un bajo porcentaje de nuestros estudiantes disfruta de beca universitaria, lo cual hace pensar que quizá algunos de los problemas que muchas veces enfrentan y que los hace abandonar los estudios podrían estar relacionados con el apoyo o no desde la universidad en ese aspecto. Un reto que se desprende de esta situación es guiar al estudiantado acerca de las fechas y los procedimientos universitarios para las solicitudes de beca, para la petición de revisión de beca en los casos en que así se requiera y promover, desde la administración, la consideración de aquellos casos que desde la unidad académica se identifiquen como prioritarios, para promover la permanencia estudiantil en la carrera y evitar factores de exclusión estudiantil. Habitualmente destacan estudiantes que son brillantes en su desempeño académico, pero que en un determinado momento manifiestan al gestor o gestoras de carrera, e incluso al profesorado, que deben retirarse de la universidad, dado que sus familias no pueden costear los estudios o incluso que no han podido entregar la documentación requerida, porque los mismos problemas económicos que enfrentan les dificulta recopilar toda la información que se les solicita en los formularios de beca. En estos casos, se hace necesaria la respuesta pronta y eficaz de la administración para atender estos procesos y evitar la pérdida de talentos, en especial si se considera el lema institucional de la "universidad necesaria".

Una acción que la subdirección de la DEB ha trabajado, desde el año anterior, es la coordinación con la trabajadora social de la Oficina de Atención Estudiantil del CIDE para atender casos de estudiantes identificados con situaciones críticas a nivel familiar y financiero, que requieren un apoyo económico urgente para continuar sus estudios y no abandonar su carrera.

Esta coordinación involucra varios aspectos, el primero es la identificación de estos estudiantes, lo cual se da por referencia del profesorado, vía carta, correo electrónico, conversación o por referencia del gestor o gestoras de carrera. Posteriormente, la subdirección analiza el caso y se contacta con las estudiantes y los estudiantes para darles una cita y, personalmente, conversar y plantearles las posibilidades de apoyo que podrín gestionarse. Este diálogo es muy relevante, pues permite comprender mejor el caso y las situaciones económico-familiares que limitan al estudiantado a 
seguir en la carrera. Por tanto, se le pregunta al estudiante si está dispuesto a avalar que se realicen las gestiones para conseguir el apoyo, pues dichos trámites requieren dar a conocer aspectos personales y familiares de cada alumna o alumno a las otras instancias de la Universidad Nacional, las que pueden dar el apoyo, dado que cuentan con ciertos recursos económicos. Además, en ocasiones, este apoyo se complementa con un acompañamiento a nivel psicológico o de orientación que se gestiona en el departamento respectivo.

Consecutivamente, se coordina con la Oficina de Atención Estudiantil (OAE), específicamente con la trabajadora social para que conozca los casos y cuente con copia de la documentación enviada por oficios a las otras instancias de la UNA y con las cuales esta trabajadora tiene un mayor contacto, por lo que les da un seguimiento a los casos y a los trámites que desde la subdirección se hayan realizado. Las instancias con las que se coordina principalmente son el Departamento de Bienestar Estudiantil y la Federación de Estudiantes (FEUNA). Este apoyo de la OAE es muy importante porque le da un seguimiento a los trámites y se logra que esas entidades lo atiendan de forma más personalizada y constante.

Cabe indicar, además, que tanto la subdirección de la DEB como la trabajadora social de la OAE señalan a los estudiantes los posibles trámites a realizar para que intenten compensar sus dificultades económicas de una forma más permanente.

De igual manera, continuando con los principales retos, es válido destacar que la mayoría de los estudiantes y las estudiantes que están cursando las carreras de la División de Educación Básica la indican como su primera opción de estudio.

Si se parte del hecho de que el estudiantado está matriculado en la carrera de su preferencia, un reto pedagógico es lograr "enamorarles" en ella, propiciar el sentido de pertenencia, fomentar las mejores prácticas para desarrollar ese gusto por la pedagogía y ofrecer opciones que promuevan su sentido de pertenencia en la unidad académica y, particularmente, con la cohorte de estudiantes que se han matriculado en cada una de las carreras que se ofrecen en este año 2012. En ese sentido, el profesorado se enfrenta al reto de promover, con los distintos cursos que ofrece, el deseo de aprender y el desarrollar las mejores aptitudes y valores hacia la formación en el campo pedagógico. Este es uno de los retos más importantes para poder aprovechar ese rasgo que identifica al estudiante de primer ingreso en este año particular.

Cabe señalar que la formación del futuro personal docente es una de las metas importantes para las universidades, razón por la cual el seguimiento de los estudiantes de primer ingreso garantiza la calidad de su educación.

Al respecto, es pertinente que la DEB mantenga, con el apoyo del gestor y gestores de carrera, la estrategia de coordinación y trabajo de nivel, pues el trabajo integrado de los profesores y las profesoras que atienden los grupos de primer ingreso, tanto en el primer como en el segundo ciclo lectivo, permite un mayor conocimiento de las particularidades del estudiantado y puede lograr potencias sus habilidades y su interés por la carrera, así como fortalecer las áreas identificadas como susceptibles de mejora. 
URL: http://www.una.ac.cr/educare

CORREO: educare@una.cr

Resulta fundamental plantear un proyecto formativo para la DEB, que apoye la formación profesional del estudiantado, a partir de aquellas debilidades y que brinde el seguimiento mediante talleres, proyectos o actividades de educación continua, u otras alternativas en las que participen académicos y académicas de la DEB.

En el plano más inmediato, es necesario plantear algunas alternativas de atención para los estudiantes y las estudiantes de la promoción 2012. Hay áreas fuertes, identificadas por los estudiantes y las estudiantes que son altamente compatibles con las características deseables en un docente tales como: la solidaridad (98\%), por el compromiso (95\%) y el saber escuchar (93\%), trabajo en equipo (87\%), la puntualidad (75\%) y liderazgo (71\%). No obstante lo indicado, hay áreas que desde la visión estudiantil son débiles y que desde nuestra posición son necesarias de atender. Entre esas se destacan las siguientes: hablar en público (65\%), habilidades deportivas (55\%), habilidades tecnológicas (48\%), redacción y ortografía (40\%, no responde $1 \%)$, habilidades artísticas (32\%), liderazgo (27\%, no responde $2 \%)$, aspectos también vinculantes con la práctica profesional docente y sumamente necesarios.

Se considera que los principales retos pedagógicos son, en primera instancia, comunicar al profesorado de cada carrera estos datos y propiciar, desde la gestión de cada carrera, la incorporación de estos aspectos en cada uno de los cursos que se imparten a lo largo de todo el proceso de formación; de manera que, el hablar en público, las habilidades tecnológicas, deportivas, la redacción y ortografía sean tratados de forma transversal. Así como también que el equipo de profesorado se comprometa con responder a las necesidades sugeridas por esta experiencia de inducción.

En segunda instancia se propone la definición de talleres específicos que puedan ser ofrecidos al estudiantado, con el criterio que la inversión en el desarrollo de este tipo de áreas, a futuro, se constituye en un elemento positivo que se verá reflejado en una mejor atención de los niños y las niñas que estos futuros docentes tendrán a su cargo.

Al respecto, se plantea desde el PDAGO la gestión de varios talleres durante este año 2012, uno desarrollado en el I Ciclo y otros a desarrollar en el II Ciclo. Se detallan a continuación: Durante el primer I Ciclo, luego de analizado el proceso de inducción y realizado el informe correspondiente, se procedió a realizar, en coordinación con la Oficina de Atención Estudiantil, el taller de Técnicas de estudio básicas para estudiantes de las carreras de la DEB. Para su desarrollo, se coordinó con una profesora o profesor de unos de los cursos del primer nivel de carrera para solicitarle el espacio de su clase para realizar el taller con los estudiantes, dicha actividad es impartida por la coordinadora y la trabajadora social de la OAE.

Para el II Ciclo 2012, el PDAGO tiene coordinado realizar un taller sobre Expresión oral para la mediación pedagógica; una actividad recreativa, y un taller sobre Habilidades tecnológicas aplicadas en la formación docente. La intención del PDAGO es reforzar las áreas susceptibles de mejora que identificó el propio estudiantado como debilidades y solicitar el apoyo del recurso humano de la DEB. 
En el caso del taller de Expresión oral y el de Habilidades tecnológicas se gestionará de dos a tres sesiones en un horario favorable para los estudiantes, de manera que las estudiantes interesadas y estudiantes interesados puedan participar.

Además, se gestionará con el académico o académica que impartirá las sesiones, los detalles de los talleres, entre ellos, fechas y duración, espacio físico (aula, laboratorio de informática), materiales, listas de asistencia, certificado de participación para el estudiantado participante, entre otros que puedan surgir.

En cuanto a la actividad recreativa, se pretende realizarla en un espacio abierto, con el apoyo de un académico de la DEB con formación en Educación Física y Recreación, de manera que organice una campaña recreativa para los estudiantes de primer ingreso, pero con posibilidad de estar abierta a los demás estudiantes regulares de la DEB.

Se organizará con el académico la fecha, hora, materiales y otros detalles que surjan de la planificación de la actividad.

Por otra parte, se destacan retos que han de ser retomados a corto y mediano plazo para favorecer el adecuado desempeño estudiantil. Entre ellas se consideran como prioritarias las siguientes:

a. Coordinar y establecer en conjunto con la Oficina de Atención Estudiantil, y por ende, con el Programa Éxito Académico, durante este año 2012 y posteriores, un cronograma de talleres que respondan y atiendan las necesidades de los estudiantes, identificadas en la ficha estudiantil según las áreas académicas.

b. Estimular las áreas fuertes que los estudiantes y las estudiantes han manifestado poseer y hacer esfuerzos por buscar alternativas de atención para las áreas que, de acuerdo con su opinión, son consideradas como deficientes.

Con las acciones propuestas anteriormente, también se responde al Modelo Pedagógico de la Universidad Nacional (Unviersidad Nacional Costa Rica, 2005) el cual destaca:

La Universidad Nacional favorece la atención integral de los estudiantes y, de manera particular, de los provenientes de sectores con carencias en sus experiencias culturales y educativas, quienes constituyen la población meta de la institución. Ello significa ofrecer las condiciones para el crecimiento personal y académico, mediante estrategias que garanticen partir de los conocimientos e ideas previas, para generar la construcción de significados y el logro de las metas establecidas en los perfiles profesionales de las carreras. (p. 8) 
URL: http://www.una.ac.cr/educare

CORREO: educare@una.cr

\section{Reflexiones finales}

Para concluir se puede afirmar que la experiencia de inducción es un primer acercamiento clave con el estudiantado y se constituye en una posibilidad para comunicar bienvenida, acogida, compartir significados y resaltar su valor en el proceso pedagógico. También, explorar opciones, propiciar situaciones de aprendizaje y comprometerse con retos que se traduzcan en oportunidades para mejorar el proceso formativo que se construye en cada una de las carreras de la División de Educación Básica.

Por otra parte, recordar que la Universidad se construye desde el saber y el sentir de las personas involucradas en el proceso, por ello, toda oportunidad de convivencia y diálogo es de gran valor, principalmente, cuando se propicia, comparte y socializa desde los primeros momentos en que estudiantado se incorpora a la institución universitaria. Así como lo afirma Maturana (2008) “La tarea de la Universidad como ámbito de convivencia, de acción y reflexión, es ampliar los espacios de acciones y reflexiones de sus estudiantes, [desde el inicio de todo proceso]" (p. 227).

Desde las propias reflexiones del equipo, también se hace una inducción para recordar que la universidad es un organismo vivo $y$, como tal, para su transformación, debe ser alimentada con insumos de todas las personas que le confieren vitalidad. Por ello, resulta medular brindar oportunidades reales para que el estudiantado ofrezca aportes desde su vida misma, sus necesidades, intereses, subjetividades y complejidad; en esencia, sus identidades.

En concordancia con las reflexiones anteriores y retomando la universidad como organismo vivo, Castillo y Espinoza (2010) indican que:

Es fundamental, que este cambio no separe al estudiantado del mundo, sino que lo incluya en el como partícipe creativo. Abandonando la ilusión de la objetividad. La complejidad está básicamente en atender la subjetividad individual y las interrelaciones que conforman la intersubjetividad. (p. 5)

\section{Referencias}

Camacho, M., Espinoza, R. A., Herrera, A., Ruiz, L. S., Segura, S. y Vásquez, E. (2011a). Programa de Desarrollo Académico y Gestión Organizacional de la División de Educación Básica. Manuscrito inédito. Heredia, Costa Rica: División de Educación Básica, Universidad Nacional.

Camacho, M., Espinoza, R. A., Herrera, A., Ruiz, L. S., Segura, S. y Vásquez, E. (2011b). Informe de Avance del Programa Académico y Gestión Organizacional. Universidad Nacional, Heredia, Costa Rica. Manuscrito inédito. Heredia, Costa Rica: Universidad Nacional.

Camacho, M., Castillo, R. A., León, H., Miranda, A., Pereira, Z. y Vásquez, E. (2012). Informe Proceso de Inducción Estudiantes Primer Ingreso 2012. Manuscrito inédito. Heredia, Costa Rica: División de Educación Básica, Universidad Nacional. 
Camacho, M., Espinoza, R. A., Herrera, A., Ruiz, L.S., Segura, S. y Vásquez, E. (2012). Informe Final del Programa de Desarrollo Académico, 2011. Manuscrito inédito. División de Educación Básica Heredia, Costa Rica: Universidad Nacional.

Castillo, R. y Espinoza, R. (marzo de 2010). La universidad como organismo vivo: De la profesión a la humanidad. Congreso Internacional de Educación Superior (CIESUP). Llevado a cabo en el Congreso de la Universidad Nacional, San José, Costa Rica.

Guevara, H. M. (enero-junio, 2009). Identidades estudiantiles, conocimiento y cultura. Percepciones de jóvenes universitarios y universitarias de Cuyo, Argentina. Revista Latinoamericana de Ciencias Sociales, Niñez y Juventud, 7(1), 209-234. Recuperado de http://redalyc.uaemex.mx/src/inicio/ArtPdfRed.jsp?iCve=77307109

Maturana, H. (2008). El sentido de lo humano. Santiago, Chile: J. C. Sáez editor.

Ocampo, E. (2007). El estudiante en la perspectiva de la pedagogía ignaciana: De la ratio a nuestra realidad. Pontificia Universidad Javeriana. Recuperado de http://www.puj.edu.co/ pedagogia/documentos.html

Pereira, Z. (noviembre, 2010). Las dinámicas interactivas en el ámbito universitario: El clima de aula. Revista Electrónica Educare 14(Extraordinario), 7-20. Recuperado de http://www. revistas.una.ac.cr/index.php/EDUCARE/article/view/1524

Universidad Nacional Costa Rica. (2005). Modelo pedagógico de la Universidad Nacional de Costa Rica. Heredia, Costa Rica: Autor. Recuperado de http://unaweb.una.ac.cr/index. php?option=com_remository\&ltemid=0\&func=startdown\&id $=141$

Venegas, M. E. (2009). ¿Cómo se forman los docentes para las escuelas en Centroamérica y República Dominicana? San José, Costa Rica: Coordinación Educativa y Cultural Centroamericana.

Cómo citar este artículo en APA:

Camacho, M., Castillo, R., León, H., Miranda, A., Pereira, Z. y Vásquez, E. (2013). Identidades estudiantiles y retos pedagógicos. Revista Electrónica Educare, 17(1), 47-65. Recuperado de http://www.revistas.una.ac.cr/index.php/EDUCARE/issue/current

Nota: Para citar este artículo en otros sistemas puede consultar el hipervínculo "Como citar el artículo" en la barra derecha de nuestro sitio web:

http://www.revistas.una.ac.cr/index.php/EDUCARE/index 